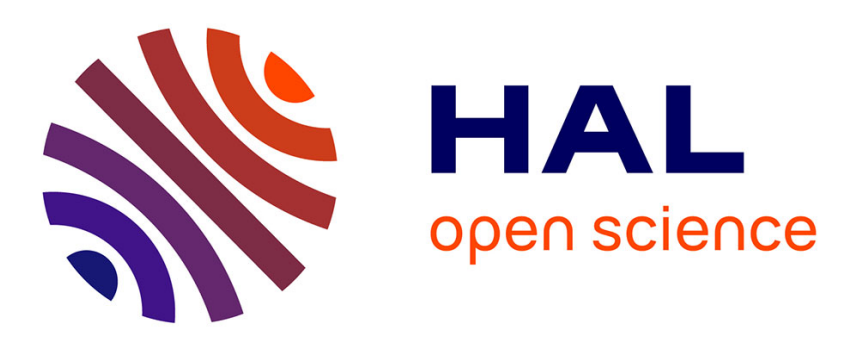

\title{
Les ingénieurs des Ponts et Chaussées face aux inondations en Roussillon (1770-1800).
}

Bertrand Desailly

\section{To cite this version:}

Bertrand Desailly. Les ingénieurs des Ponts et Chaussées face aux inondations en Roussillon (17701800).. Revue Géographique des Pyrénées et du Sud-Ouest, 1989, Sud-Ouest vers 1750 - vers 1875, 60 (3), pp.329-343. 10.3406/rgpso.1989.3162 . hal-03161721

\section{HAL Id: hal-03161721 \\ https://hal.science/hal-03161721}

Submitted on 8 Mar 2021

HAL is a multi-disciplinary open access archive for the deposit and dissemination of scientific research documents, whether they are published or not. The documents may come from teaching and research institutions in France or abroad, or from public or private research centers.
L'archive ouverte pluridisciplinaire HAL, est destinée au dépôt et à la diffusion de documents scientifiques de niveau recherche, publiés ou non, émanant des établissements d'enseignement et de recherche français ou étrangers, des laboratoires publics ou privés. 


\section{Les ingénieurs des Ponts et Chaussées face aux inondations en} Roussillon (1770-1800)

\section{Bertrand Desailly}

\section{Citer ce document / Cite this document :}

Desailly Bertrand. Les ingénieurs des Ponts et Chaussées face aux inondations en Roussillon (1770-1800). In: Revue géographique des Pyrénées et du Sud-Ouest, tome 60, fascicule 3, 1989. Sud-Ouest vers 1750 - vers 1875. pp. 329-343; doi : https://doi.org/10.3406/rgpso.1989.3162

https://www.persee.fr/doc/rgpso_0035-3221_1989_num_60_3_3162

Fichier pdf généré le 06/04/2018 


\begin{abstract}
The civil engineering corps confronting the floods in Roussillon. During the last decades of the Ancien Régime the province of Roussillon was hit by a number of major floods. Civil engineers from the Highways Department discovered that the practice of clearing forest land in the mountains was destabilising slopes and causing considerable alluviation. However their only reaction was to draw up plans to straighten the principal rivers on the flood plain, to invite riverside residents to strengthen the banks by planting trees and, locally, to organise the construction of dykes.
\end{abstract}

\title{
Resumen
}

Los ingenieros de puentes y caminos frente a las inundaciones del Rosellón. Durante los últimos decenios del Antiguo Regimen, la provincia del Roselló sufrió una sucessión de inundaciones catastróficas. Al reflexionar sobre el origen de estas acontecimientos, los ingenieros de Puentes y Caminos los achacaron a las roturaciones efectuadas en la sierra, con la subsiguiente desestalización de las vertientes y fuertes aportaciones aluviales. Sin ambargo su intervención había de limitarse a la llanura. Alli elaboran planes de rectificación de los principales ríos. Alli incitan a los habitantes a fortalecer las riberas con árboles. Alli amprenden, localmente, la construcción de diques.

\section{Résumé}

Au cours des dernières décennies de l'Ancien Régime, la province du Roussillon connaît une série d'inondations catastrophiques. Amenés à réfléchir sur l'origine de ces événements, les ingénieurs des Ponts et Chaussées mettent en cause les défrichements pratiqués dans la montagne, facteur de déstabilisation des versants et d'un important alluvionnement. Leur intervention se limite cependant à la plaine, où ils tracent des plans d'alignement des principaux cours d'eau, invitent les riverains à fortifier les berges par des plantations, et dirigent localement des travaux d'endiguement. 
REVUE GÉOGRAPHIQUE DES PYRÉNÉES ET DU SUD-OUEST TOME 60, FASC. 3, pp. 329-343, Toulouse, 1989.

\section{Les ingénieurs des Ponts et Chaussées face aux inondations en Roussillon (1770-1800)}

par Bertrand DESAILLY*

Au début du mois de novembre 1763, François de Lescure, ingénieur des Ponts et Chaussées de la province du Roussillon, remonte la vallée du Tech afin de constater les dommages causés par les pluies diluviennes survenues quinze jours plus tôt, les 16 et 17 octobre. Le rapport minutieux qu'il nous a laissé (1), l'évaluation des dégâts qui fut alors faite, nous incitent à voir en cet événement un précédent probable de la crue extraordinaire d'octobre 1940, référence obligée pour qui s'intéresse aux catastrophes naturelles dans cette partie des Pyrénées. Des dizaines de moulins et de granges emportées, des forges détruites, leurs réserves de minerai et de charbon de bois entraînées par les eaux, et, de part et d'autre du Tech, d'immenses épandages de blocs et de graviers : le haut Vallespir devait offrir certainement un spectacle affligeant, qu'on aurait pu trouver à quelques nuances près dans les bassins voisins de la Têt et de l'Agly. Dans les seuls environs de Prats-de-Mollo, neuf personnes avaient péri noyées.

Après un milieu de siècle plutôt calme, l'aiguat (2) d'octobre 1763 inaugure ainsi de façon dramatique une longue série d'inondations plus ou moins graves, qui se succèdent dans les Pyrénées catalanes jusqu'à la fin de la période révolutionnaire. Quelques dates se déta-

* Agrégé de géographie, Assistant à l'Institut Daniel Faucher, Université de Toulouse-Le Mirail, 31058 Toulouse cedex (CIMA-U.A. 366 CNRS).

(1) Archives départementales des Pyrénées-Orientales (A.D. 66), 1 C 1078.

(2) Un aiguat désigne, en Roussillon, un abat d'eau exceptionnel et l'inondation concomitante. 
chent, associées chaque fois un peu partout à des champs, des prés ou des vignes dévastées: octobre 1766, septembre et décembre 1772, novembre et décembre 1777, janvier 1787, mars 1790, décembre 1795(3). Il n'est guère surprenant dans ce contexte que les ravages des eaux apparaissent en bonne place dans les cahiers de doléances des communautés du Roussillon (4). La question préoccupe pareillement l'administration locale, avant comme après 1789 , qui tente sans grand succès d'imposer des plans de redressement des cours d'eau et d'organiser les riverains en syndicats de défense des berges. La Révolution n'apporte à ce sujet aucune modification sensible du contenu des discours et des attitudes. En effet, au-delà du pittoresque de certaines formulations - aux crues d'eau de l'Ancien Régime font suite les extumescences révolutionnaires - il est clair que la même inspiration guide les règlements des derniers intendants, les décisions du directoire du nouveau département, ou les arrêtés des préfets du début de l'Empire. On la trouverait sans trop de peine dans les nombreux rapports, mémoires et projets que rédigent à l'époque les ingénieurs des Ponts et Chaussées.

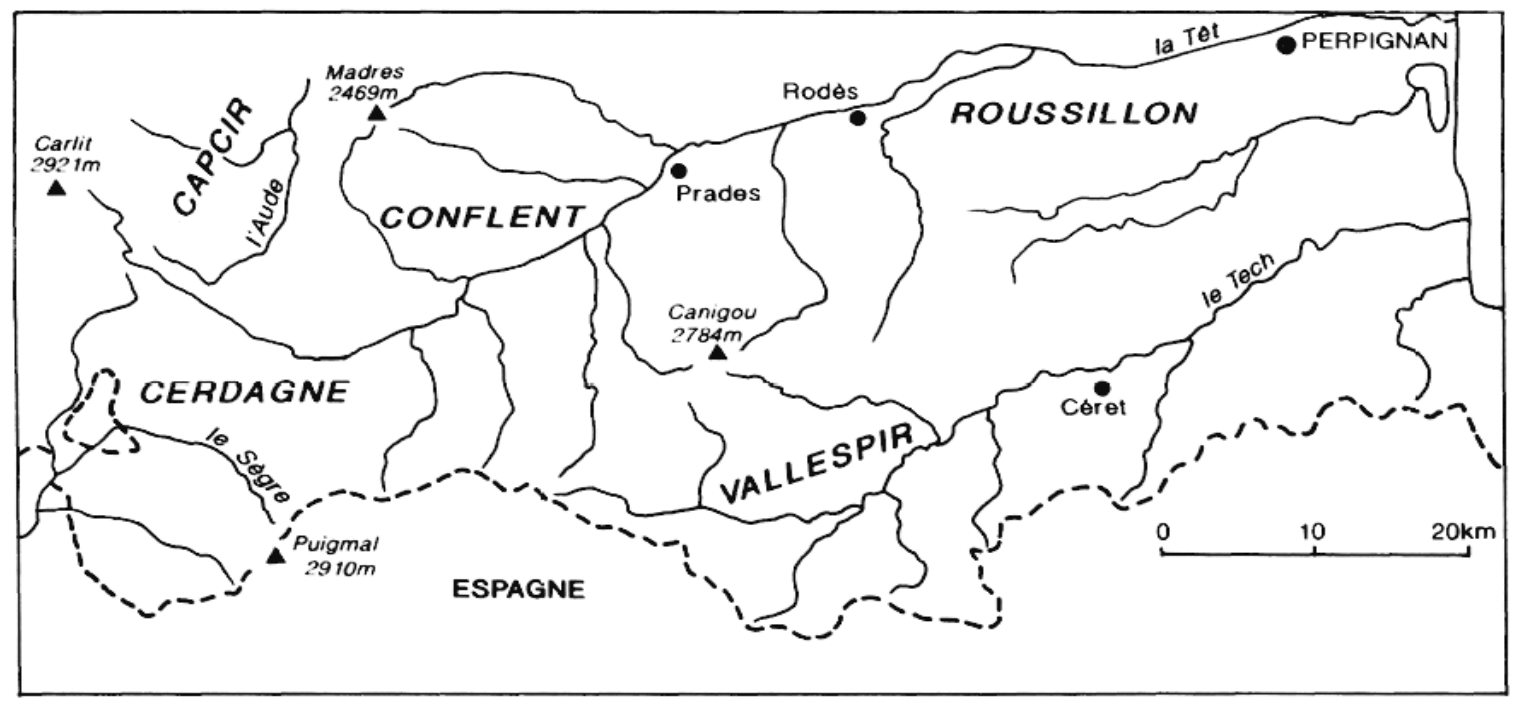

FIG. 1

Roussillon : croquis de localisation.

(3) On trouvera un recensement des principales inondations survenues en Roussillon au cours du XVIII ${ }^{\circ}$ siècle dans: GUIRAUD DE SAINT-MARSAL, Mémoire sur les inondations occasionnées par les crues de la Têt et de la Basse aux environs de Perpignan, Société Agricole, Scientifique et Littéraire des PyrénéesOrientales, tome 10, 1856, pp. 223-273.

(4) Sur les 132 cahiers de doléances conservés des communautés du Roussillon, 48 font état des dommages causés par les débordements des rivières. Ces cahiers ont été publiés par Etienne FrenAY, Cahiers de doléances de la province du Roussillon (1789), Perpignan, Direction des Services d'Archives, 1979, 427 p. 
Arrivés dans la province en 1750, les ingénieurs des Ponts et Chaussées ont progressivement et non sans conflits pris la place occupée jusqu'alors par leurs homologues du Génie militaire dans le domaine de l'aménagement des cours d'eau (5). Ces derniers avaient conduit dans la première moitié du siècle d'importants travaux fluviaux, en particulier l'endiguement de la Têt aux abords de Perpignan, sur environ un kilomètre, achevé vers 1735 (6). Mais leur intervention gardait un caractère ponctuel, sous-tendue surtout par la nécessité de préserver les fortifications de la capitale du Roussillon. Si la protection contre les eaux de Perpignan et de ses alentours demeure, jusqu'à la fin du XVIII* siècle, la principale priorité, les ingénieurs des Ponts et Chaussées étendent notablement leur champ d'intervention, surveillant par exemple la rectification d'un méandre de la Têt à Prades, entre 1780 et 1788. Mais surtout, l'un des leurs, Kolly de Montgazon, s'interroge pour la première fois vers 1775 sur les causes de ces inondations qui paraissent affecter de plus en plus souvent les terroirs de la plaine. Au premier rang des responsables sont désignés les défrichements pratiqués et encouragés dans la montagne.

\section{Propos sur les débordements des rivières du Roussillon}

\section{Le déterminisme naturel.}

Si les ingénieurs croient discerner, dans le dernier tiers du XVIII ${ }^{\circ}$ siècle, une augmentation de la fréquence et de la gravité des crues - encore l'unanimité n'est-elle pas réalisée sur ce point - ils se refusent à la mettre cn relation avec une quelconque péjoration climatique. Cette hypothèse rencontre pourtant selon eux un certain assentiment populaire, dont le fondement irrationnel fait qu'ils n'y accordent guère de crédit : le climat se serait modifié depuis le tremblement de terre de Lisbonne. Que le Roussillon soit exposé à recevoir de copieuses averses, voilà qui est avéré; mais tel est le lot commun de toutes les provinces du sud du royaume, et rien ne permet d'affirmer que ces pluies étaient moins abondantes dans la première moitié du siècle: "[l'inondation du 7 décembre 1772] fut accompagnée et occasionnée par une de ces pluies du midi de la France qui versent quelquefois deux à trois pouces de hauteur d'eau et davantage par heure sur chaque toise carrée de la surface de terrain qui la reçoit.

(5) Sur la mise en place de l'administration des Ponts et Chaussées en Roussillon, voir : Nelly Pousthomis, Sylvie CAUCANas et Philippe Rosset, Les routes en Roussillon, milieu XVII' siècle - milieu $X I X^{e}$ siècle, Perpignan, Direction des Services d'Archives, 1986, $136 \mathrm{p}$.

(6) Voir à ce sujet: Bertrand Desailly, L'aménagement du lit de la Têt à Perpignan; un exemple de travaux contre les crues au dix-huitième siècle, $B u l l$. ass. géogr. franç., Paris, 1990, 1, pp. 23-34. 
Ces pluies sont inconnues à Paris et dans le nord de l'Europe. Mais la Provence, le Languedoc, le Pays de Foix, le Roussillon, le Béarn en furent les victimes cette même année 1772 »(7). Pierre Poeydavant, subdélégué général de l'intendance, dans le tableau détaillé qu'il dresse en 1778 du Roussillon et du Pays de Foix (8), insiste davantage sur le rôle des conditions topographiques : "De petites plaines entourées de montagnes, excepté du côté de la mer, reçoivent toutes les eaux qui tombent sur les hauteurs ou s'y rassemblent en nature de neige. La proximité de ces montagnes, la rapidité des pentes et les matières plus ou moins pesantes que les eaux détachent en s'écoulant contribuent à leur donner une force à laquelle il est difficile d'opposer avec succès quelque résistance ». Climat et relief conjuguent donc leurs effets pour rendre le Roussillon vulnérable aux ravages des eaux.

En cette fin du XVIII' siècle, les ingénieurs qui dirigent l'aménagement de la Têt à proximité de Perpignan sont confrontés au problème posé par un alluvionnement accéléré. Toutes les solutions sont envisagées pour que le courant enlève ces encombrants dépôts : augmenter la pente du lit et réduire sa largeur entre les digues, creuser des canaux, supprimer tous les obstacles susceptibles de provoquer des atterrissements, etc. Le radier du Pont de pierre qui assure le franchissement du fleuve est enfoui vers 1770 sous un mètre cinquante de sables et de graviers - on ignore malheureusement à quel moment il se trouvait dégagé. Il serait certes hasardeux de conclure, à partir de cette seule observation, à l'existence d'une phase de remblaiement alluvial en Roussillon à la fin de l'époque moderne. D'autant que les travaux d'endiguement du fleuve à l'amont du pont menés par les militaires entre 1725 et 1735 ont pu localement influer grandement sur la vitesse d'écoulement des eaux. On comprend cependant par là que les ingénieurs aient rendu l'exhaussement continuel des lits responsable de débordements toujours plus nombreux. De fait, le profil transversal en toit de la vallée de la Têt à l'aval de Perpignan est éminement favorable au développement d'inondations très étendues, pour peu que le niveau du lit approche celui des terres environnantes. Depuis la rive septentrionale du fleuve jusqu'à l'Agly, les altitudes ne cessent de diminuer, et les villages de cette région - la Salanque se sont trouvés maintes fois sous les eaux dans les dernières décennies de l'Ancien Régime. La lettre écrite par l'intendant Guillaume de Bon au Contrôleur général des finances, après la crue de décembre 1772, ne contient d'exagération que dans la réunion des eaux de la Têt et du Tech, laquelle ne peut être réalisée : "Le Tech, la Têt et l'Agly débouchent dans la mer à peu de distance l'une de l'autre; et

(7) "Mémoire d'éclaircissement sur l'objet des représentations de plusieurs habitants de Perpignan qui ont été inondés lors d'une crue de la rivière de la Basse au mois de décembre 1772 ", par M. de Fourcroy. Archives de l'Inspection du Génie (A.I.G.), article 8, section 1.

(8) Le mémoire de Pierre Poeydavant a été publié par la Société Agricole, Scientifique et Littéraire des Pyrénées-Orientales (volume 95, 1987, 398 p.). 
comme dans les grandes crues d'eau, elles s'épanchent dans les campagnes à droite comme à gauche, elles se joignent et forment en approchant des côtes des nappes d'eau d'un volume immense (...). Il résulte de cet exposé que non seulement les propriétaires des fonds voisins des rivières et des torrents, mais encore les tenanciers dont les biens sont situés dans l'intérieur des terres sont continuellement exposés à des réparations qui absorbent une bonne partie de leurs revenus " (9). Mais comment réduire ces apports incessants de matériaux qui comblent les lits?

\section{Les défrichements en accusation.}

1777 est une année sombre pour la province du Roussillon, accablée par les aléas climatiques. Les froids tardifs du printemps, qui ruinent la récolte de seigle dans les hauts pays du Capcir et du Conflent, sont suivis à l'automne par des inondations catastrophiques de tous les cours d'eau. La Têt déborde à deux reprises à Perpignan; elle y occasionne de gros dégâts : la digue Orry en rive gauche du fleuve (10), le Pont des Eaux vives et la Chaussée du Vernet, qui prolongent le Pont de pierre en direction de Narbonne, sont partiellement renversés. Peu de temps après, l'intendant invite l'ingénieur des Ponts et Chaussées, Kolly de Montgazon, et le directeur des fortifications Desclaisons à se concerter, afin de proposer des solutions pour empêcher définitivement le retour de tels désastres. Les deux ingénieurs consignent le résultat de leur réflexion dans un mémoire, qui est envoyé pour examen à l'Assemblée des Ponts et Chaussées, à Paris. Selon eux, la racine du mal réside dans les défrichements inconsidérés entrepris en montagne, principalement depuis 1766 où un édit royal les a encouragé par des exemptions fiscales. Sur les versants nouvellement dénudés, l'érosion se serait emballée, entraînant un accroissement de la charge des cours d'eau et par conséquent des dépôts. A cela, il faudrait ajouter un gonflement beaucoup plus brutal des torrents, en raison d'une fonte plus rapide de la neige - on admet alors que les eaux de crue viennent pour l'essentiel de la fusion nivale : "il est aisé de sentir que les arbres ou les arbustes dont les montagnes étaient couvertes autrefois garantissaient la neige de l'action subite de la chaleur, elle ne fondait que par degrés, les eaux retenues par les différents obstacles qu'elles rencontraient n'étaient pas de sitôt réunies "(11). Le caractère néfaste des défrichements étant donc bien établi, il conviendrait d'y mettre un terme, faute de

(9) A.I.G., article 5, section 1, § 21.

(10) Philibert Orry fut intendant du Rousillon de 1727 à 1729. C'est à cette époque que fut achevée la grande digue en rive gauche de la Têt, à laquelle il laissa son nom.

(11) A.D. 66, 1 C 1214. 
quoi le succès de nouveaux travaux d'endiguement de la Têt serait des plus hypothétiques.

Kolly de Montgazon exprime là une opinion assez largement partagée, semble-t-il, par les ingénieurs de son époque, du moins par ceux qui exercent dans une région montagneuse. On la retrouvera, par exemple, étayée par un raisonnement solide, dans l'Essai sur la théorie des torrents et des rivières que J.A. Fabre écrit en 1797 (12). A Versailles ou à Paris, on adopte toutefois une position généralement moins tranchée. Car les défrichements concourent à accroître la richesse du pays et sont le signe visible de sa bonne santé démographique. Il n'est donc pas souhaitable de les interdire absolument, sauf dans quelques cas bien spécifiques. Le rapport tout en nuances que Pierre Marie Jérôme Trésaguet rend à l'Assemblée des Ponts et Chaussées, après avoir étudié le mémoire reçu des ingénieurs du Roussillon, est à cet égard significatif. L'extension des surfaces cultivées en montagne est-elle à la source d'une recrudescence des inondations? "Quoique cette allégation paraisse probable, on ne peut cependant porter aucun jugement sur l'option d'arrêter les défrichements pour rétablir les choses dans leur premier état inculte et désert (...). L'administrateur de la province peut seul par une juste combinaison des intérêts particuliers avec ceux du public se rendre compte du parti le plus avantageux à prendre »(13).

L'administrateur de la province est précisément enclin à suivre les recommandations de ses ingénieurs. La coïncidence, à quelques années près, entre la publication de l'édit royal favorisant les essartages et le début d'une série de crues dévastatrices, était en effet troublante. Raymond de Saint-Sauveur, nommé intendant du Roussillon en 1778, annonce aussitôt qu'il va "solliciter une loi du prince qui prohibe à l'avenir tout défrichement dans la montagne, et règle le sort de ceux qui ont été faits »(14). Une déclaration royale du 8 avril 1779 répond à cette demande, mais il faut supposer qu'elle ne fut pas suivie d'un grand effet. En 1791, les membres du directoire du département des Pyrénées-Orientales, dans une série d'Observations sur les débordements des rivières, reprennent en une langue plus imagée, mais sans la modifier, l'argumentation développée quinze ans plus tôt par Kolly de Montgazon : "Il est notoire à quiconque a de l'expérience qu'une pluie de vingt jours (sic) et au-delà n'était pas suffisante anciennement pour faire dégorger nos rivières, tandis qu'actuellement, une chute de 24 heures suffit pour les faire déborder (...). Les graviers, les rocs des montagnes étant à découvert et ne se trou-

(12) J.-A. FABRE, Essai sur la théorie des torrents et des rivières, contenant les moyens les plus simples d'en empêcher les ravages, d'en rétrécir le lit, etc., Paris, Bidault, 1797.

(13) Archives Nationales (A.N.), F 14169.

(14) Règlement de Raymond de Saint-Sauveur du 25 novembre 1778, instituant deux syndicats de défense des berges de la Têt à l'aval de Perpignan, A.N., F 14574 et A.D. 66, 1 C 1215. 
vant plus contenus par des friches et des arbustes, il est naturel qu'ils suivent le cours des eaux et se précipitent avec elles dans leur entrepôt qui est le canal des rivières; et le lit de celles-ci se trouvant par cet accident de niveau au moins avec les terres voisines, elles doivent nécessairement s'épandre à la moindre crue et prendre en espace ce qu'elles ont perdu en profondeur » (15).

Il serait évidemment intéressant de connaître l'étendue réelle des défrichements effectués en Roussillon dans les dernières décennies de l'Ancien Régime. De 1767 à 1782, les bureaux de l'intendance adressent chaque année à Versailles un état des terres nouvellement mises en culture. Celles-ci représenteraient, sur l'ensemble de la période considérée, une superficie totale de 3600 éminates, soit environ 2100 hectares. Cette statistique embryonnaire soulève davantage de questions qu'elle ne permet d'en résoudre. Les chiffres ont pu être exagérés, afin de mettre en avant la vitalité économique de la province, et inversement, combien d'essartages, effectués dans des zones marginales, ont échappé à toute comptabilité ? Quelle est la part de champs et de vignes - le vin connaît localement une sérieuse crise de mévente après 1770 - abandonnés au bout de quelques années ? Il est surtout étrange, à lire ces états, que la quasi totalité des défrichements recensés aient eu lieu dans la seule viguerie de Roussillon et Vallespir. La viguerie de Conflent et Capcir aurait été épargnée par le mouvement, en raison, nous dit-on dans une note, d'entraves formées par les seigneurs. Or, le Conflent correspond précisément, à peu de choses près, au bassin moyen et supérieur de la Têt, là même où les ingénieurs voient l'érosion se déchaîner. Une chose paraît certaine : la moyenne vallée de la Têt, en amont de Prades, est à l'époque assez intensément mise en valeur. Tel plan de Villefranche de Conflent, dessiné vers 1765, nous montre la soulane dominant la place-forte couverte de vignes, soutenues par des murettes. Palassou, qui visite les lieux une dizaine d'années plus tard, confirme cette impression: " $\mathbf{A}$ un quart de lieue après Prades, on commence à pénétrer dans les hautes montagnes, la vallée se rétrécit considérablement (...) mais le voyageur est moins effrayé par la pente rapide des montagnes que ravi de voir une quantité prodigieuse de vignes sur la rive gauche de la Têt qui, toute hérissée de rochers, semblait devoir se refuser aux travaux des cultivateurs (...). Les vignes continuent d'embellir les bords de la Têt jusqu'aux environs d'Olette; on ne voit pas, dans toute la chaîne, de montagnes qui soient aussi bien cultivées » (16). Mais les ingénieurs mettent moins en cause ces cultures en terrasses que celles entreprises au milieu des vacants, à l'issu d'un rapide brûlis, et délaissées après deux ou trois récoltes, sans que des murettes de soutènement aient été construites. Il est permis de penser que ce procédé,

(15) A.D. 66, L 1108.

(16) Palassou, Essai sur la minéralogie des Monts Pyrénées, Paris, Didot jeune, 1781. 
fort répandu dans les Préalpes du sud selon Raoul Blanchard (17), n'était pas non plus inconnu dans les Pyrénées catalanes.

\section{Le plan de lutte des ingénieurs}

\section{Distinguer plaine et montagne.}

Seuls les défrichements en montagne sont jugés dangereux. On doit continuer de leur donner libre cours dès lors qu'ils s'opèrent en terrain peu accidenté. Ces principes admis, l'administration provinciale va s'efforcer de procéder à une division en deux zones de chaque bassin fluvial. Pierre Poyedavant, dans son mémoire composé en 1778 (supra), est l'un des premiers à s'y attacher: "On pourrait ainsi déterminer dans le Roussillon des points fixes au-dessus desquels le nouveau règlement [relatif aux défrichements] serait mis à exécution; ces points serviraient de division entre la plaine et la montagne, et peut-être seraient-ils bien établis, savoir au Pont de Céret pour la partie du Vallespir, et au terroir de Rodès pour celle du Conflent. On ne fait point ici mention des points supérieurs du cours de l'Agly, lesquels se trouvent en Languedoc "(18). Le découpage suggéré est assez fruste: Poeydavant demeure en partie prisonnier de frontières administratives préexistantes. Si le Pont de Céret franchit le Tech non loin de l'endroit où celui-ci débouche dans la plaine, le terroir de Rodès marque en fait surtout la séparation entre les vigueries de Roussillon et de Conflent. Le cas du chaînon des Albères, drainé par des cours d'eau se jetant dans le Tech à l'aval de Céret, n'est pas évoqué. Que dire du bassin de l'Agly, à cheval sur deux généralités, où toute intervention est par conséquent exclue ? Avec la déclaration royale du 8 avril 1779 sur les défrichements en Roussillon, on s'achemine vers une zonation beaucoup moins tributaire d'anciennes délimitations, et davantage de la topographie. C'est ainsi que les essartages sont également prohibés sur les reliefs pourtant peu élevés qui s'étendent au nord de la Têt, depuis Rodès à l'amont jusqu'à Força-Réal à l'aval, non loin de Perpignan.

La volonté de reboiser la montagne, après y avoir interdit toute nouvelle mise en culture, n'est jamais très clairement affirmée. On compte davantage sur les capacités de la nature pour assurer, petit

(17) Raoul Blanchard réservait le terme d'essartage à ces cultures temporaires sur brûlis, en lesquelles il voyait: "le chancre qui a rongé la végétation des pentes et dont l'action corrosive, tantôt atténuée, tantôt exaspérée, s'est faite sentir depuis une lointaine antiquité jusqu'au XIX ${ }^{*}$ siècle ». Le lien entre ce type de pratique et la torrentialité ne faisait pour lui pas de doute. Voir par exemple : "Déboisement et reboisement dans les Préalpes françaises du sud *, Revue de Géographie alpine, Grenoble, tome 32, 1944, pp. 335-388.

(18) Mémoire de Pierre Poeydavant sur la province du Roussillon, op. cit., p. 314. 
à petit, la reconstitution d'un couvert végétal fourni. Il convient de souligner l'égale valeur de protection des versants attribuée à la forêt et à la pelouse. Le déboisement, s'il n'a d'autre but que d'ouvrir des pâturages, n'est pas considéré comme réellement néfaste. On ne trouvera, dans les mémoires et rapports des ingénieurs, aucune attaque en règle contre les bergers des grands troupeaux transhumants, ni même contre les charbonniers du Vallespir. Vers la fin du siècle toutefois, les premiers signes d'un changement d'attitude se font jour. Le reboisement commence à être envisagé, selon des modalités quelque peu fantaisistes il est vrai : "Il conviendrait (...) d'exciter de nouvelles plantations, au moins à une distance donnée, dans les terrains proches des rivières, et surtout là où les ravins se dégorgent (...). Ces plantations formeraient des espèces d'estacades qui arrêteraient les terres, graviers et les rochers qui vont se précipiter dans le lit des rivières et les comblent »(19). J.A. Fabre était plus convaincant, qui proposait à la même époque d'alterner, suivant les courbes de niveau, bandes cultivées et bandes boisées (20). On reste encore loin, en tout cas, du grand dessein des forestiers dans la seconde moitié du siècle suivant.

\section{Projets et grands travaux.}

Sinon pour y réglementer les défrichements, les ingénieurs n'envisagent pas d'intervenir directement dans la montagne : la correction des torrents n'est pas à l'ordre du jour. C'est donc en plaine qu'ils vont trouver matière à exercer leur art. Le programme est simple: il s'agit d'assigner un lit fixe aux rivières, d'en fortifier les berges par des plantations et, ponctuellement, par des digues beaucoup plus onéreuses : seuls les abords de Perpignan bénéficient d'un tel traitement.

La démarche préliminaire à tout travail de cette nature consiste à réaliser des plans d'alignement. Tous les ingénieurs, ou peu s'en faut, s'attellent successivement à cette tâche, qu'on peut bien regarder comme un exercice de style, d'où des considérations d'ordre esthétique ne sont pas totalement absentes. L'esprit qui doit les animer est clairement signifié dans un règlement publié en 1778 par l'intendant Raymond de Saint-Sauveur : "Tracer dès la chute des montagnes un alignement le plus direct possible, qui puisse former à la rivière un lit d'une largeur égale dans son cours, et dont chaque côté soit défendu par une plantation de profondeur suffisante pour régler le cours des eaux, sans trop les contraindre »(21). La Têt surtout est alors l'objet de toutes les attentions. Les ingénieurs militaires furent

(19) A.D. 66, L 1108.

(20) J.-A. FABRE, Essai sur la théorie des torrents et des rivières, op. cit.

(21) A.N., F 14574 et A.D. 66, 1 C 1215. 


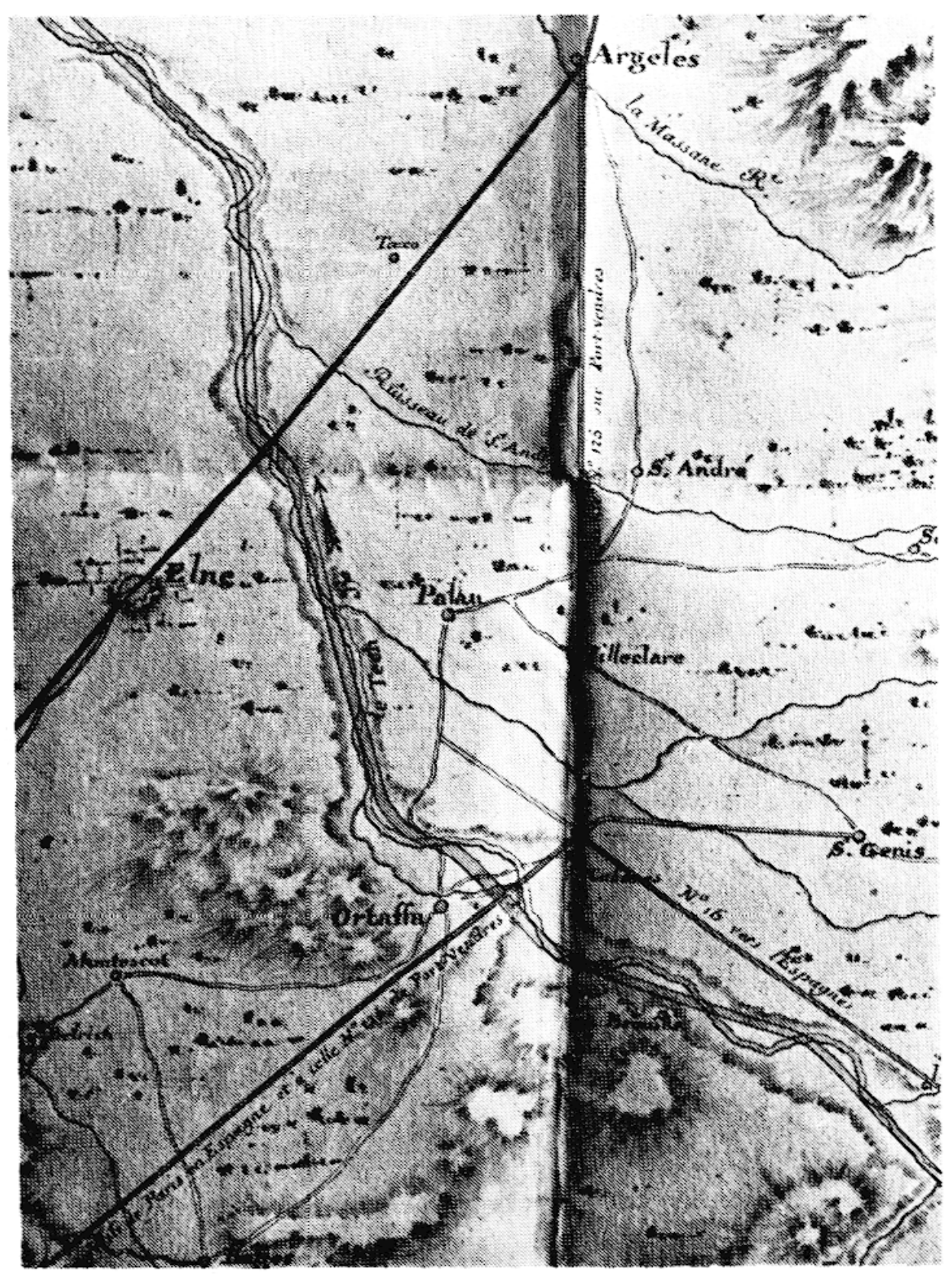

Fig. 2

"Plan de différents projets de communications en 1803 pour la route $n^{\circ} 16$ de première classe de Paris en Espagne (...) où l'on a tracé le redressement à faire à la rivière du Tech aux frais des propriétaires riverains ».

1803, (Archives nationales, F 14888 ). 
les premiers à en dresser des plans d'alignement, sur des distances relativement courtes (quelques kilomètres), dans la première moitié du XVIII" siècle (22). Ils se montraient de fervents adeptes de la ligne droite ou légèrement brisée. Leurs projets, trop abstraits du tracé réel du fleuve, se révélaient totalement inapplicables. Quelques décennies plus tard, les ingénieurs des Ponts et Chaussées n'hésitent pas à faire usage d'amples courbes, pour l'élégance de la forme autant peut-être que pour ne pas s'écarter trop des sinuosités du cours d eau. Parallèlement, l'accord se fait sur la largeur à donner au lit du fleuve : 40 toises ( 80 mètres), et à chacun des deux francsbords : 24 toises (48 mètres). Ces francs-bords doivent être plantés en saules, osiers ou peupliers par les riverains, qui ont également l'obligation de maintenir ces plantations à l'état de taillis, en les coupant par tiers chaque année. On compte ainsi raffermir les berges, les élever progressivement en provoquant des atterrissements et amortir la violence des courants de crue.

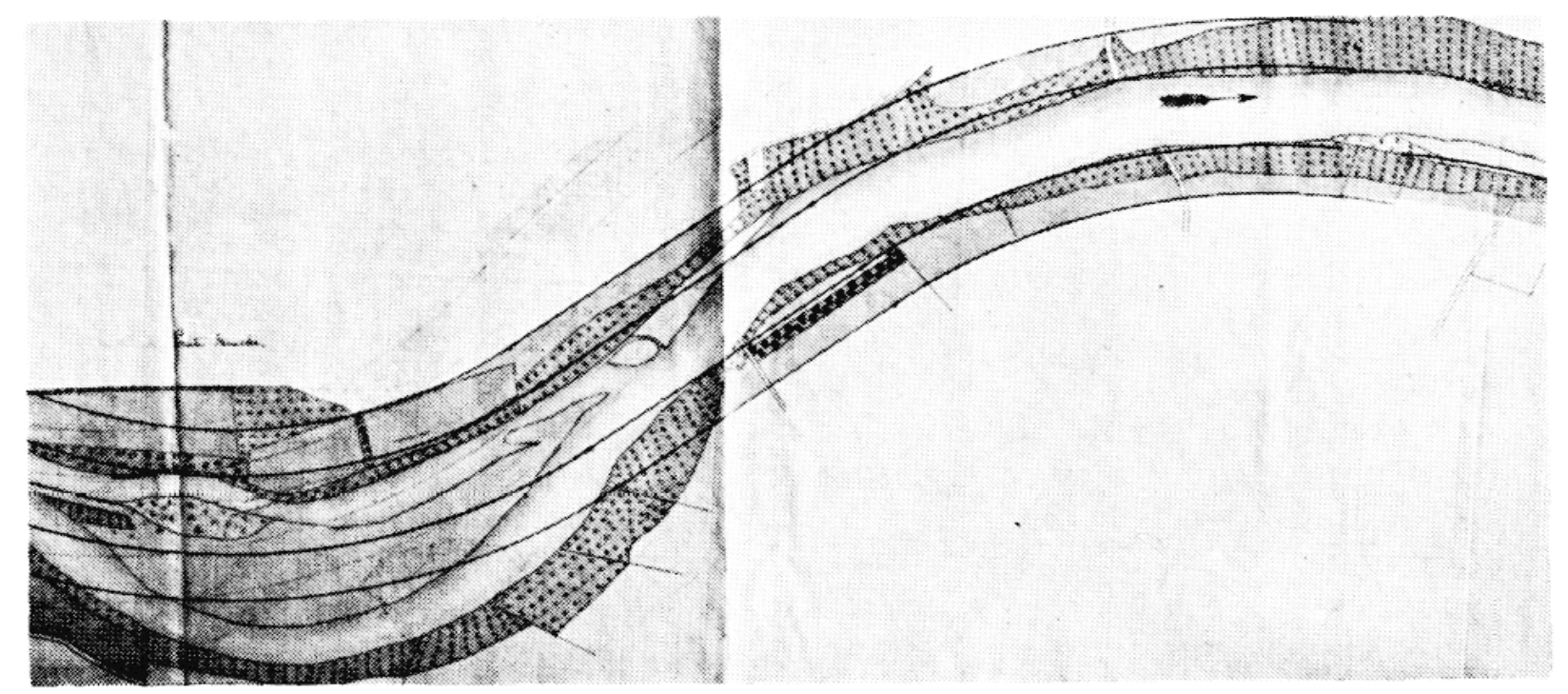

FIG. 3

"Plan géométrique de la rivière de la Têt depuis le Pont de pierre de Perpignan jusqu'à la mer \%, par L'Eveillé, 1816 (détail), (Archives nationales, F 14 10081).

Une fois le plan d'alignement matérialisé sur le terrain par des piquets peuvent débuter les travaux de redressement du cours d'eau. Les obstacles qui s'élèvent alors sont tels que l'entreprise s'arrête généralement à ce stade. Le premier réside dans les contestations vigoureuses qui s'élèvent inéluctablement entre les tenanciers des deux

(22) Voir Bertrand Desailly, Un exemple de travaux de protection contre les crues au XVIII' siècle, op. cit. 
bords au sujet du tracé adopté. Les ingénieurs ne peuvent jouer le rôle d'arbitre : la confiance qu'on leur accorde est fort limitée depuis que l'un d'entre eux, Lescure, fut accusé en 1765, à la faveur d'un achat de terrain qu'il fit le long de la Têt, d'avoir empiété sur l'alignement tracé par son prédécesseur. Le second obstacle est d'ordre financier : dans l'esprit des ingénieurs comme de l'administration, la charge des travaux incombe entièrement aux propriétaires riverains, qui ne peuvent escompter aucune aide en la matière. Or, cette charge est loin d'être négligeable, puisqu'il faut rectifier des méandres, guider les eaux dans le nouveau lit par des batardeaux et des canaux creusés dans les alluvions, arracher les arbres et les buissons qui l'encombrent. Seules des associations syndicales sont à même de récolter des sommes suffisantes. Elles existent déjà au XVIII ${ }^{\mathbf{e}}$ siècle, rassemblant les riverains d'un seul bord, mais leur statut est des plus flou et leur fonctionnement loin d'être satisfaisant. Des rôles sont mis en recouvrement de façon épisodique, au hasard des dégâts causés par telle ou telle crue et qu'il est urgent de réparer. Les intendants puis les préfets vont tenter de leur donner une base solide. Le règlement de Raymond de Saint-Sauveur du 25 novembre 1778, l'arrêté du préfet Charvet du 15 thermidor an VIII, sont les étapes qui conduisent à l'ordonnance royale du 20 mai 1818, portant règlement d'administration publique pour la rivière de la Têt, qui organise à l'aval de Perpignan de façon définitive deux syndicats de défense des berges. Chacun de ces deux syndicats, dont le périmètre recouvre l'ensemble des terrains menacés par les débordements du fleuve, réunit désormais les propriétaires de rive droite et de rive gauche (23).

A Perpignan même, la situation est sensiblement différente, puisque l'aménagement de la Têt est financé par des fonds publics. Le fleuve est endigué depuis 1735, mais les travaux menés par les militaires sont loin d'avoir donné satisfaction. Les digues, édifiées à l'aide des sables et graviers extraits du fond du lit, se montrent excessivement fragiles. Lors de chaque crue importante, de larges brèches sont ouvertes dans la digue Orry, que les eaux contournent en outre par le nord, envahissant le Champ de Mars et bousculant la chaussée de la route de Narbonne. A l'issue des inondations de l'automne 1777, deux projets sont avancés pour remédier à cet état de fait. Le premier, conçu par le directeur des fortifications Desclaisons, vraisemblablement plus à son affaire dans la construction de remparts, consiste à substituer à la digue Orry un mur de maçonnerie, renforcé de place en place par des éperons. Le projet de l'ingénieur des Ponts et Chaussées Montgazon porte sur un prolongement des digues et sur leur renforcement par un revêtement en perré; il est finalement adopté en 1780 ,

(23) Pour plus de détails sur ces aspects juridiques, on se reportera, par exemple, à Adrien SAISSET, La plantation en bois-taillis dans francs-bords de la rivière de la Têt d'après 't'ordonnance royale du 20 mai 1818, Perpignan, Commission syndicale de la Rivière de la Têt, 1928, 32 p. 
non sans que le devis initial, s'élevant à quelques 800000 livres, n'ait été réduit de moitié. La somme est encore énorme, face aux 30 ou 40000 livres dont disposent chaque année les Ponts et Chaussées du Roussillon. Aussi a-t-on recours à un mode de financement original : 500000 livres sont affectées à la réalisation des ouvrages, ainsi qu'accessoirement à l'entretien des routes, fournis moitié par l'Etat, moitié par le produit d'un impôt spécial levé dans la province et créé à cette occasion.

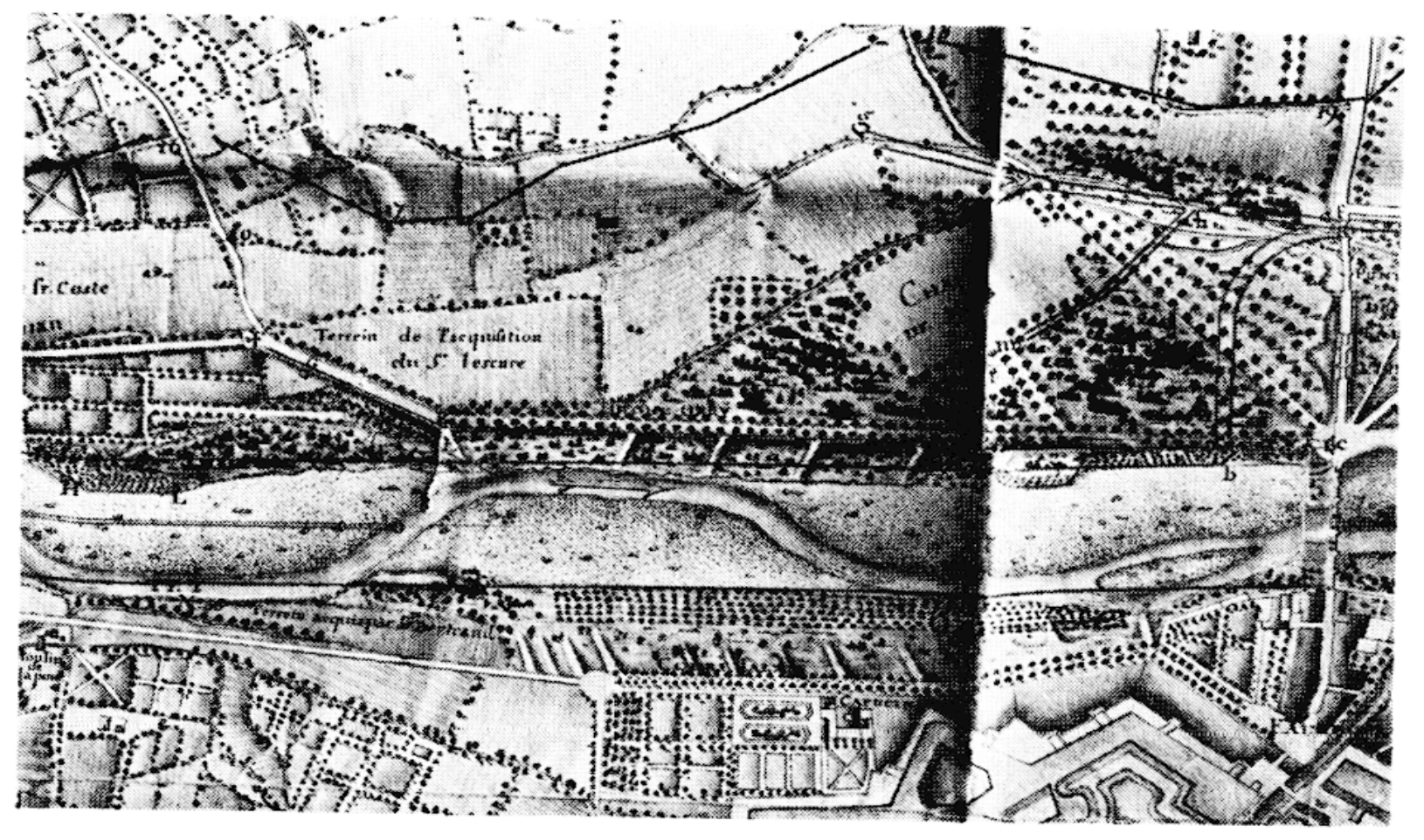

Fig. 4

"Plan topographique d'une partie du cours de la rivière de la Têt depuis au-dessus du Mas Générer jusqu'au-dessous des ponts de Perpignan ", par

La Têt est contenue en amont du Pont de pierre de Perpignan entre les digues Orry et Jallais, toutes deux protégées par des épis et des plantations.
(Archives nationales, F 14 169).

Au moment où éclate la Révolution, le projet Montgazon est loin d'avoir été mené à bien, quoique le devis ait été dépassé. Dès lors, et durant un quart de siècle, les digues de la Têt à Perpignan ne vont plus recevoir semble-t-il qu'un entretien minimum. La crue catastrophique d'octobre 1814 n'aura aucun mal à ouvrir une nouvelle brèche dans la digue Orry, au perré complètement ruiné. 
En cette fin du $\mathrm{XVIII}^{e}$ siècle, la conception qu'ont les ingénieurs de la lutte contre les inondations n'est peut-être pas aussi novatrice qu'elle ne le paraît de prime abord. Il serait exagéré d'y voir l'esquisse d'un projet d'aménagement global des bassins-versants, comme on en verra dans la seconde moitié du siècle suivant. Car à vrai dire, les seuls travaux envisagés dans le lit des rivières doivent s'opérer en plaine. Sans doute les ingénieurs du Roussillon ont-ils éprouvé un sentiment d'impuissance face à des cours d'eau au régime si peu pondéré, réduits à un filet d'eau la plupart du temps, gonflés démesurément par les pluies d'automne et de printemps, et véhiculant alors une quantité énorme de matériaux. Sans doute ont-ils pu mesurer l'efficacité limitée d'ouvrages hydrauliques conçus pour d'autres sortes de rivières, la difficulté de fixer les lits. Ils ont recherché les moyens de réduire les engravements, et en ont rendu responsables les défrichements dans les montagnes. Mais la montagne elle-même est exclue du projet d'aménagement. Y interdire les défrichements il fait peu de doute que cette interdiction ne fut guère respectée c'est entrevoir la possibilité de façonner plus tranquillement le lit des fleuves, en plaine. Le peu d'intérêt que les ingénieurs accordent aux montagnards victimes des éléments est d'ailleurs significatif. Les inondations sont moins graves selon eux dans les vallées étroites, parce que la nappe d'eau ne peut s'y étaler: l'expédition de Lescure dans le haut Vallespir, après l'aiguat d'octobre 1763, n'a donc guère porté ses fruits. Pour l'ingénieur du siècle des Lumières, selon Antoine Picon, "le territoire produit en quelque sorte l'aménagement comme la réalisation de ses aspirations les plus profondes » (24). A l'évidence, à la veille de la Révolution, la montagne nord catalane n'est pas encore de ces territoires là.

(24) Antoine PIcon, Architectes et ingénieurs du siècle des Lumières, Marscillc, Parenthèses, 1988, p. 220. 
RÉSUMÉ. - Au cours des dernières décennies de l'Ancien Régime, la province du Roussillon connaît une série d'inondations catastrophiques. Amenés à réfléchir sur l'origine de ces événements, les ingénieurs des Ponts et Chaussées mettent en cause les défrichements pratiqués dans la montagne, facteur de déstabilisation des versants et d'un important alluvionnement. Leur intervention se limite cependant à la plaine, où ils tracent des plans d'alignement des principaux cours d'eau, invitent les riverains à fortifier les berges par des plantations, et dirigent localement des travaux d'endiguement.

SUMmary. - THE CIVIL ENGINEERING CORPS CONFRONTING THE FLOODS IN ROUSSILLON. During the last decades of the Ancien Régime the province of Roussillon was hit by a number of major floods. Civil engineers from the Highways Department discovered that the practice of clearing forest land in the mountains was destabilising slopes and causing considerable alluviation. However their only reaction was to draw up plans to straighten the principal rivers on the flood plain, to invite riverside residents to strengthen the banks by planting trees and, locally, to organise the construction of dykes.

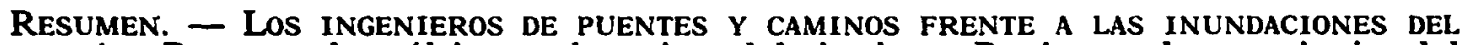
Rosellón. Durante los últimos decenios del Antiguo Regimen, la provincia del Roselló sufrió una sucessión de inundaciones catastróficas. Al reflexionar sobre el origen de estas acontecimientos, los ingenieros de Puentes y Caminos los achacaron a las roturaciones efectuadas en la sierra, con la subsiguiente desestalización de las vertientes y fuertes aportaciones aluviales. Sin ambargo su intervención había de limitarse a la llanura. Alli elaboran planes de rectíficación de los principales ríos. Alli incitan a los habitantes a fortalecer las riberas con árboles. Alli amprenden, localmente, la corstrucción de diques.

Mots-Clés. - Pyrénées, Pyrénées-orientales, Roussillon, inondations, défrichements en montagne, ingćnicurs des Ponts et Chaussées, aménagement des cours d'eau, XVIII' sieccle. 